\title{
Update on the Surgical Management of Fuchs Endothelial Corneal Dystrophy
}

\author{
Andrea L. Blitzer · Kathryn A. Colby
}

Received: June 29, 2020 / Published online: August 25, 2020

(C) The Author(s) 2020

\section{ABSTRACT}

Fuchs endothelial corneal dystrophy (FECD) is the most common posterior corneal dystrophy and the leading indication for corneal transplantation in the United States. FECD is slowly progressive, and patients develop gradual corneal endothelial decompensation, eventually resulting in failure of the endothelium to maintain corneal deturgescence. Medical management consists of topical hyperosmotic agents to facilitate dehydration of the cornea, but surgical intervention is often required to regain corneal clarity. The surgical management of FECD has evolved over the past two decades as corneal transplantation techniques have allowed for more selective keratoplasty and replacement of only the diseased layers of the cornea. Prior surgical management consisted of penetrating keratoplasty (PK) that carried significant intraoperative risks associated with "open sky" as well as postoperative risks of graft rejection, wound dehiscence, postoperative astigmatism, and prolonged visual rehabilitation. In the past 15 years, endothelial

Digital Features To view digital features for this article go to https://doi.org/10.6084/m9.figshare.12783635.

A. L. Blitzer · K. A. Colby $(\bowtie)$

Department of Ophthalmology and Visual Science, University of Chicago Medical Center, Chicago, IL, USA

e-mail: kcolby@bsd.uchicago.edu keratoplasty (EK) has become the treatment of choice for endothelial disease, significantly reducing the risks associated with the surgical treatment of FECD. Here we discuss the current surgical management of FECD, including the introduction of Descemet stripping only (DSO), and highlight future investigative efforts.

Keywords: Corneal

transplantation; Descemetorhexis; Descemet membrane endothelial keratoplasty; Descemet stripping; Descemet stripping automated endothelial keratoplasty; Fuchs dystrophy

\section{Key Summary Points}

\section{Why carry out this study?}

This review summarizes the surgical management of Fuchs endothelial corneal dystrophy (FECD).

\section{What was learned from the study?}

The surgical management of FECD has evolved over the past two decades as corneal transplantation techniques have allowed for more selective keratoplasty. 
The current preferred surgical management of FECD is endothelial keratoplasty, which carries risks associated with the need for indefinite immunosuppression with topical steroids.

Descemet stripping only (DSO) involves removal of the diseased endothelium and guttae without the placement of any donor graft.

DSO has a very high rate of success, but requires careful patient selection.

\section{INTRODUCTION}

Fuchs endothelial corneal dystrophy (FECD), the most common endothelial dystrophy, is the leading indication for corneal transplantation in the United States, accounting for more than one third of the transplants performed in 2019 [1]. In the past decade, corneal transplantation, and specifically the surgical management of FECD, has changed drastically [2]. For many years, the only transplantation option for visually debilitating FECD consisted of penetrating keratoplasty (PK). In 2012, endothelial keratoplasty (EK) surpassed PK as the most commonly performed keratoplasty procedure, and selective keratoplasty became the mainstay of surgical treatment for FECD $[1,3]$. While the majority of FECD patients requiring keratoplasty continue to undergo EK, recent findings in the lab and in multiple clinical case series have suggested a role for descemetorhexis without keratoplasty, known as Descemet stripping only (DSO). Here we review the pathogenesis of FECD, compare DSO with EK as treatment options, and highlight future directions for the management of FECD. This article is based on previously conducted studies and does not contain any studies with human participants or animals performed by any of the authors.

\section{OVERVIEW OF FUCHS ENDOTHELIAL CORNEAL DYSTROPHY}

The corneal endothelium is a neural crestderived monolayer of cells, halted in the G1 phase of the cell cycle and thought to not divide after birth, though in vitro studies have revealed a potential for cell division [4]. The endothelium plays a critical role in corneal homeostasis by maintaining deturgescence, a requirement for corneal transparency. The corneal stroma has a robust capacity to swell [5], which is counteracted by the endothelial cells. As water passively moves into the stroma during the active transport of nutrients, the endothelial cells provide both a passive barrier to excessive aqueous humor influx and maintain an active pump mechanism to transport fluid out of the cornea. Deturgescence requires a sufficient number of endothelial cells, and when the density of corneal endothelial cells drops below about 500 cells $/ \mathrm{mm}^{2}$, as may occur in FECD, the endothelial pump function fails and the cornea becomes edematous.

At birth, the cornea has the highest density of corneal endothelial cells at about 4000 cells/ $\mathrm{mm}^{2}$. As the cornea ages, the cell density will decrease to about 2500 cells $/ \mathrm{mm}^{2}$ in a healthy adult cornea [6]. Because of the limited proliferative potential of the corneal endothelium, cells must migrate to take the place of neighboring cells that are lost due to injury or aging, resulting in decreased cell density, increased variation in cell size, and loss of the typical hexagonal architecture. Despite these changes, normal corneal aging does not typically result in endothelial cell failure leading to edema. However, in diseased states such as in FECD, the number of endothelial cells may drop below the critical density necessary to maintain the endothelial pump function, resulting in a loss of corneal clarity.

The hallmark finding in FECD is the presence of central guttae, excrescences of Descemet membrane (DM), on the posterior cornea. Though guttae may be found in up to $4 \%$ of patients, few of them will develop the corneal edema associated with FECD [7]. FECD is slowly 
progressive, with clinically apparent guttae usually presenting in the fourth decade of life, and significant reduction in vision requiring intervention often not occurring until decades later. Though visual acuity can remain good even in advanced FECD, glare and diurnal variations in vision may become disabling. The Visual Function and Corneal Health Status instrument (V-FUCHS) is a self-administered questionnaire that can be used in the clinic to effectively measure visual disability in patients with FECD [8]. Guttae may directly degrade vision quality by producing light scatter $[9,10]$. In addition to this direct effect on vision, in vitro studies have shown that guttae may create a toxic environment that contributes to endothelial cell loss that may in turn allow for the development of more guttae and promote a cycle of endothelial decompensation [11].

\section{SURGICAL MANAGEMENT FOR FUCHS ENDOTHELIAL CORNEAL DYSTROPHY}

Current medical therapy for FECD-related vision loss is limited to decreasing corneal edema with topical hyperosmotic drops and ointment, and often surgical intervention is required to regain corneal clarity. When the only available transplantation option for FECD was $\mathrm{PK}$, the threshold for surgical intervention was high, and patients were followed conservatively until they developed advanced disease. Nearly a century after Zirm performed the first PK in 1905, Melles described a method for transplanting only the posterior layers of the cornea while leaving the host anterior cornea intact [12]. Posterior lamellar keratoplasty (PLK), also termed deep lamellar endothelial keratoplasty (DLEK) [13], involved replacing the host posterior stroma, DM, and endothelium with a donor button through a sclerocorneal incision and has paved the way for modern EK. With EK, the risk of surgical intervention including graft rejection, prolonged visual rehabilitation, infection, and postoperative astigmatism has decreased significantly, and the risk associated with "open sky" is eliminated. Patients are now undergoing transplantation earlier in the disease course, guided by visual symptoms and clinical findings [2].

\section{Descemet Stripping Automated Endothelial Keratoplasty}

Descemet stripping automated EK (DSAEK) is the most commonly performed EK in the United States. In DSAEK, a descemetorhexis is performed on the host cornea to remove the diseased endothelium and DM. Typically the descemetorhexis is large (at least $8 \mathrm{~mm}$ ) but may be smaller depending on surgeon preference. This tissue is then replaced by a donor posterior lamellar button that is approximately $100-200 \mu \mathrm{m}$ thick and contains endothelium, $\mathrm{DM}$, and a thin layer of stroma. Handling of the transplant graft is facilitated by the attached stromal lamellae, and an air bubble is placed in the anterior chamber to promote graft adherence.

DSAEK, described in its first iteration by Melles et al. in [14] and later modified by Gorovoy [15], represented a major breakthrough in the surgical management of posterior corneal disease. Though graft rejection remains a risk, it is significantly decreased compared to PK [16]. Additionally, visual outcomes are better, though acuity may be limited by the interface between host and donor stroma and posterior corneal higher-order aberrations resulting from graft thickness variations. Visual rehabilitation is quicker than in $\mathrm{PK}$, and there is less risk of postoperative astigmatism. Newer modifications to DSAEK have created thinner grafts. Ultrathin DSAEK (UT-DSAEK) (60-90 $\mu \mathrm{m}$ grafts) and nanothin DSAEK (NT-DSAEK) (grafts $50 \mu \mathrm{m}$ or thinner) have been shown to have improved visual outcomes with faster visual recovery than standard DSAEK [17-20].

\section{Descemet Membrane Endothelial Keratoplasty}

With the development of Descemet membrane endothelial keratoplasty (DMEK) in 2006, Melles et al. again changed the landscape of corneal transplantation [21, 22]. DMEK allows for transplantation of only DM and 
endothelium to the host cornea after descemetorhexis, with grafts as thin as $10 \mu \mathrm{m}$.

DMEK requires greater surgical skill than DSAEK. Without the adherent stroma, the elastic nature of the tissue causes the graft to fold into a scroll or double scroll, and there is a steep learning curve to developing unfolding techniques. The natural scroll causes the endothelium to face outward, but alternatively the graft may be manipulated to an endothelialinward trifold configuration [23]. Though this increases the time for graft preparation, it may minimize surgical time and endothelial cell loss. A comparison of grafts inserted in the endothelium-outward scroll or endotheliuminward trifold showed comparable graft failure rates, suggesting that the insertion method ultimately depends on surgeon preference [24]. Other modifications to DMEK include the use of a femtosecond laser to create a more precise descemetorhexis [25], hemi- and quarter-DMEK to increase the availability of donor tissue $[26,27]$, and eye bank preparation of prestripped and preloaded tissue to decrease surgical time. Despite its technical challenges, the number of DMEK procedures has increased each year in the United States, even as the number of DSAEK procedures has decreased [1]. Compared to DSAEK and PK, DMEK has the least risk of graft failure and results in the fastest visual recovery $[28,29]$.

\section{Descemet Stripping Only}

EK has produced favorable outcomes for patients with FECD in the United States; however it is not without risk; and even DMEK carries the possibility of graft rejection if topical corticosteroid immunosuppression is stopped [30-32]. Additionally, there remains a worldwide shortage of donor corneas for transplantation procedures. For these reasons, there is an interest in developing a treatment for FECD that does not require placement of a graft.

The endothelium of patients with FECD may be capable of self-regeneration. Ex vivo studies of human corneal buttons have shown that endothelial cells, even those from older donors, retain the ability to undergo mitosis [6].
Further, peripheral endothelial cells appear to have a higher potential for mitotic activity [6]. Clinically, numerous reports of inadvertent loss of endothelium and DM in patients with FECD have resulted in spontaneous resolution of corneal edema [33-43].

Encouraged by these findings, Borkar et al. published a case series of FECD patients managed with deliberate Descemet stripping only (DSO) [44]. DSO involves creating a small (4-5 $\mathrm{mm}$ ) descemetorhexis to remove the diseased endothelium and guttae without the placement of any donor graft. In this series of 13 eyes in 11 patients, ten eyes had restoration of corneal clarity and at least $20 / 20$ vision in all eyes without macular pathology. The remaining three patients underwent subsequent uneventful DMEK that resulted in corneal clearing [45].

Review of this and other case series may reveal why DSO is an effective treatment for some, but not all, FECD patients [46, 47]. Advanced disease appears to have a poor prognosis for corneal clearing with DSO. Patients with pachymetry greater than $625 \mu \mathrm{m}$ had a reduced rate of corneal clearance [44]. The presence of guttae extending into the periphery of the cornea may also reduce the likelihood of successful DSO, as there is an observed trend that a larger descemetorhexis is more likely to have prolonged corneal clearing or fail to clear without subsequent EK. Given that the peripheral endothelial cells need to repopulate the central denuded cornea, there is likely a limit to the area that they are able to cover. Overall, multiple recent case series of DSO with careful patient selection have shown excellent success, with corneal clearing in up to $100 \%$ of patients [48-50].

\section{ADVANTAGES OF DESCEMET STRIPPING ONLY}

There are many advantages to performing DSO as the primary surgery in the management of FECD. The absence of a donor tissue obviates the need for indefinite immunosuppression with topical steroids to prevent graft rejection. It also provides a possible surgical procedure in regions where there is a shortage of donor 
corneas. Unlike DMEK, which is a technically difficult surgery with a steep learning curve, DSO is a relatively simple procedure that utilizes techniques well known to corneal surgeons.

\section{DISADVANTAGES OF DESCEMET STRIPPING ONLY}

The optimal patient for DSO is still unknown. DSO requires a healthy population of peripheral endothelial cells, and is therefore unlikely to be successful in the setting of advanced FECD with peripheral guttae and decreased peripheral endothelial cell density. A peripheral endothelial cell count lower than 1800 cells $/ \mathrm{mm}^{2}$ has been shown to be associated with a lack of corneal clearance following DSO [51]. DSO is only suited to FECD, where disease starts centrally and is related to endothelial dysfunction, in contrast to other causes for endothelial decompensation, such as pseudophakic bullous keratopathy (PBK) that causes endothelial depletion affecting the entire endothelial layer including the periphery. Because DSO does not replace the endothelium at the time of surgery, there is a longer visual recovery while the endothelium repopulates enough to maintain corneal deturgescence. In contrast, DMEK demonstrates significantly faster corneal clearance and would likely be more appropriate in a patient who cannot tolerate extended periods of decreased vision. Patients need to be counseled that, if the cornea fails to clear after DSO, they may require an EK. As DSO is a newer procedure, long-term follow-up is limited. Recently published 5-year data showed sustained corneal clarity and visual acuity [52]; however, further studies will be needed to determine the longterm viability of the procedure, specifically the risk of late endothelial cell loss and delayed failure.

\section{FUTURE DIRECTIONS}

Patients fall into three categories after DSO: rapid responders, slow responders, and nonresponders. While case series suggest that lack of peripheral endothelial reserve, the size of the descemetorhexis, and the preoperative pachymetry may be predictive of corneal clearance after DSO, more work is needed to determine the optimal patients for DSO [51].

Recently, the use of topical Rho-associated kinase (ROCK) inhibitors, used in the treatment of glaucoma, has shown promise as an adjunctive therapy to DSO. ROCK inhibitors have been shown to hasten corneal clearance in animal studies, and have been used for the treatment of FECD in conjunction with endothelial removal with a cryoprobe in four patients $[53,54]$. The use of a ROCK inhibitor after DSO was able to rescue two slow responders [48], and has been shown to increase cell density and hasten corneal clearance when compared to DSO without ROCK inhibitor therapy [50].

Current randomized, placebo-controlled studies aimed at investigating the use of ROCK inhibitors after DSO are underway. ROCK inhibitors are also being evaluated in the absence of DSO to determine whether there is any potential for corneal clearance with medical therapy alone, though the current understanding of the pathogenesis of FECD suggests that this may have limited utility.

There have been exciting advancements in the use of cultured endothelial cells for corneal disease. In animal studies, cells cultured from a donor corneal endothelium have been shown to self-organize and function normally when injected, in conjunction with a ROCK inhibitor, into the anterior chamber [55]. Recently, intracameral injection of cultured endothelial cells supplemented with a ROCK inhibitor was shown to successfully treat $\mathrm{PBK}$, resulting in sustained corneal clarity and improved visual acuity 2 years after treatment [56]. No allogeneic rejection has been observed.

\section{CONCLUSIONS}

FECD is the most common endothelial dystrophy and the leading indication for corneal transplantation in the United States. Medical treatment of FECD is limited to topical hyperosmotic agents, and with the excellent risk-benefit profile of modern corneal transplantation, patients often elect to undergo 
Table 1 Surgical techniques for the management of Fuchs endothelial corneal dystrophy

\begin{tabular}{llc}
\hline Technique & Advantages & Disadvantages \\
\hline DSAEK & Eliminates "open & Requires indefinite \\
sky" risk & immunosuppression \\
compared to & Acuity may be limited \\
PK & by host-donor stroma \\
& Less postoperative & interface and higher- \\
& astigmatism & order aberrations \\
than PK & \\
DMEK & Improved visual & Technically difficult to \\
& outcomes & perform \\
& Fastest corneal & Graft rejection remains \\
& clearance & a risk \\
DSO & No introduction & Requires careful patient \\
& of donor tissue & selection
\end{tabular}

DSAEK descemet stripping automated endothelial keratoplasty, DMEK descemet membrane endothelial keratoplasty, DSO descemet stripping only, $P K$ penetrating keratoplasty

surgical intervention when FECD becomes visually debilitating (Table 1 ).

The current preferred surgical management of FECD is EK, which has shown excellent rates of corneal clearance. Though the rate of graft rejection in EK, particularly in DMEK, is low, there remains a risk of rejection with discontinuation of a topical corticosteroid. DSO is a technically simple and effective means of treating FECD without the need for donor tissue. This low-risk procedure can be performed in combination with cataract surgery. Recent studies have shown increased success of DSO when an adjunctive topical ROCK inhibitor is used. Continued research into the use of ROCK inhibitors following DSO is underway, and determination of the optimal patient characteristics for successful DSO warrants further investigation.

\section{ACKNOWLEDGEMENTS}

Funding. No funding or sponsorship was received for this study or publication of this article.

Authorship. All named authors meet the International Committee of Medical Journal Editors (ICMJE) criteria for authorship for this article, take responsibility for the integrity of the work as a whole, and have given their approval for this version to be published.

Disclosures. Andrea L. Blitzer and Kathryn A. Colby have nothing to disclose.

Compliance with Ethics Guidelines. This article is based on previously conducted studies and does not contain any studies with human participants or animals performed by any of the authors.

Open Access. This article is licensed under a Creative Commons Attribution-NonCommercial 4.0 International License, which permits any non-commercial use, sharing, adaptation, distribution and reproduction in any medium or format, as long as you give appropriate credit to the original author(s) and the source, provide a link to the Creative Commons licence, and indicate if changes were made. The images or other third party material in this article are included in the article's Creative Commons licence, unless indicated otherwise in a credit line to the material. If material is not included in the article's Creative Commons licence and your intended use is not permitted by statutory regulation or exceeds the permitted use, you will need to obtain permission directly from the copyright holder. To view a copy of this licence, visit http://creativecommons.org/licenses/by$\mathrm{nc} / 4.0 /$. 


\section{REFERENCES}

1. Eye Bank Association of America (EBAA). 2019 Eye banking statistical report. Washington, DC: Eye Bank Association of America; 2020.

2. Sarnicola C, Farooq AV, Colby K. Fuchs endothelial corneal dystrophy: update on pathogenesis and future directions. Eye Contact Lens. 2019;45(1): $1-10$.

3. Güell JL, El Husseiny MA, Manero F, Gris O, Elies D. Historical review and update of surgical treatment for corneal endothelial diseases. Ophthalmol Ther. 2014;3(1-2):1-15.

4. He Z, Campolmi N, Gain P, et al. Revisited microanatomy of the corneal endothelial periphery: new evidence for continuous centripetal migration of endothelial cells in humans. Stem Cells. 2012;30(11):2523-34.

5. Kwok LS, Klyce SD. Theoretical basis for an anomalous temperature coefficient in swelling pressure of rabbit corneal stroma. Biophys J. 1990;57(3):657-62.

6. Joyce NC. Proliferative capacity of the corneal endothelium. Prog Retin Eye Res. 2003;22(3): 359-89.

7. Krachmer JH, Purcell JJ, Young CW, Bucher KD. Corneal endothelial dystrophy. A study of 64 families. Arch Ophthalmol. 1978;96(11):2036-9.

8. Wacker K, Mclaren JW, Kane KM, Patel SV. Corneal optical changes associated with induced edema in Fuchs endothelial corneal dystrophy. Cornea. 2018;37(3):313-7.

9. Watanabe S, Oie Y, Fujimoto $\mathrm{H}$, et al. Relationship between corneal guttae and quality of vision in patients with mild Fuchs' endothelial corneal dystrophy. Ophthalmology. 2015;122(10):2103-9.

10. Oie Y, Watanabe S, Nishida K. Evaluation of visual quality in patients with Fuchs endothelial corneal dystrophy. Cornea. 2016;35(Suppl 1):S55-S58.

11. Kocaba V, Katikireddy KR, Gipson I, Price MO, Price FW, Jurkunas UV. Association of the Gutta-induced microenvironment with corneal endothelial cell behavior and demise in Fuchs endothelial corneal dystrophy. JAMA Ophthalmol. 2018;136(8): 886-92.

12. Melles GR, Eggink FA, Lander F, et al. A surgical technique for posterior lamellar keratoplasty. Cornea. 1998;17(6):618-26.
13. Terry MA, Ousley PJ. Replacing the endothelium without corneal surface incisions or sutures: the first United States clinical series using the deep lamellar endothelial keratoplasty procedure. Ophthalmology. 2003;110(4):755-64.

14. Melles GR, Wijdh RH, Nieuwendaal CP. A technique to excise the descemet membrane from a recipient cornea (descemetorhexis). Cornea. 2004;23(3):286-8.

15. Gorovoy MS. Descemet-stripping automated endothelial keratoplasty. Cornea. 2006;25(8): 886-9.

16. Koenig SB, Covert DJ, Dupps WJ, Meisler DM. Visual acuity, refractive error, and endothelial cell density 6 months after descemet stripping and automated endothelial keratoplasty (DSAEK). Cornea. 2007;26(6):670-4.

17. Dickman MM, Kruit PJ, Remeijer L, et al. A Randomized multicenter clinical trial of ultrathin descemet stripping automated endothelial keratoplasty (DSAEK) versus DSAEK. Ophthalmology. 2016;123(11):2276-84.

18. Madi S, Leon P, Nahum Y, et al. Five-year outcomes of ultrathin descemet stripping automated endothelial keratoplasty. Cornea. 2019;38(9): 1192-7.

19. Kurji KH, Cheung AY, Eslani M, et al. Comparison of visual acuity outcomes between nanothin descemet stripping automated endothelial keratoplasty and descemet membrane endothelial keratoplasty. Cornea. 2018;37(10):1226-311.

20. Tourabaly M, Chetrit Y, Provost J, et al. Influence of graft thickness and regularity on vision recovery after endothelial keratoplasty. Br J Ophthalmol. 2019.

21. Melles GR. Posterior lamellar keratoplasty: DLEK to DSEK to DMEK. Cornea. 2006;25(8):879-81.

22. Melles GR, Ong TS, Ververs B, van der Wees J. Preliminary clinical results of descemet membrane endothelial keratoplasty. Am J Ophthalmol. 2008;145(2):222-7.

23. Busin M, Leon P, Scorcia V, Ponzin D. Contact lensassisted pull-through technique for delivery of trifolded (endothelium in) DMEK grafts minimizes surgical time and cell loss. Ophthalmology. 2016;123(3):476-83.

24. Price MO, Lisek M, Kelley M, Feng MT, Price FW. Endothelium-in versus endothelium-out insertion with descemet membrane endothelial keratoplasty. Cornea. 2018;37(9):1098-101. 
25. Pilger D, Von Sonnleithner C, Bertelmann E, Maier $A B$, Joussen AM, Torun N. Exploring the precision of femtosecond laser-assisted descemetorhexis in Descemet membrane endothelial keratoplasty. BMJ Open Ophthalmol. 2018;3(1):e000148.

26. Lam FC, Baydoun L, Dirisamer M, Lie J, Dapena I, Melles GR. Hemi-descemet membrane endothelial keratoplasty transplantation: a potential method for increasing the pool of endothelial graft tissue. JAMA Ophthalmol. 2014;132(12):1469-73.

27. Zygoura V, Baydoun L, Ham L, et al. Quarter-descemet membrane endothelial keratoplasty (Quarter-DMEK) for Fuchs endothelial corneal dystrophy: 6 months clinical outcome. Br J Ophthalmol. 2018;102(10):1425-30.

28. Price MO, Giebel AW, Fairchild KM, Price FW. Descemet's membrane endothelial keratoplasty: prospective multicenter study of visual and refractive outcomes and endothelial survival. Ophthalmology. 2009;116(12):2361-8.

29. Chamberlain W, Lin CC, Austin A, et al. Descemet endothelial thickness comparison trial: a randomized trial comparing ultrathin descemet stripping automated endothelial keratoplasty with descemet membrane endothelial keratoplasty. Ophthalmology. 2019;126(1):19-26.

30. Allan BD, Terry MA, Price FW, Price MO, Griffin NB, Claesson M. Corneal transplant rejection rate and severity after endothelial keratoplasty. Cornea. 2007;26(9):1039-42.

31. Anshu A, Price MO, Price FW. Risk of corneal transplant rejection significantly reduced with Descemet's membrane endothelial keratoplasty. Ophthalmology. 2012;119(3):536-40.

32. Price MO, Scanameo A, Feng MT, Price FW. Descemet's membrane endothelial keratoplasty: risk of immunologic rejection episodes after discontinuing topical corticosteroids. Ophthalmology. 2016;123(6):1232-6.

33. Braunstein RE, Airiani S, Chang MA, Odrich MG. Corneal edema resolution after "descemetorhexis". J Cataract Refract Surg. 2003;29(7):1436-9.

34. Patel DV, Phang KL, Grupcheva CN, Best SJ, Mcghee CN. Surgical detachment of Descemet's membrane and endothelium imaged over time by in vivo confocal microscopy. Clin Exp Ophthalmol. 2004;32(5):539-42.

35. Zvi T, Nadav B, Itamar K, Tova L. Inadvertent descemetorhexis. J Cataract Refract Surg. $2005 ; 31(1): 234-5$.
36. Pan JC, Eong KGA. Spontaneous resolution of corneal oedema after inadvertent 'descemetorhexis' during cataract surgery. Clin Exp Ophthalmol. 2006;34(9):896-7.

37. Agarwal A, Jacob S, Agarwal A, Agarwal S, Kumar MA. Iatrogenic descemetorhexis as a complication of phacoemulsification. J Cataract Refract Surg. 2006;32(5):895-7.

38. Balachandran C, Ham L, Verschoor CA, Ong TS, Van der Wees J, Melles GR. Spontaneous corneal clearance despite graft detachment in descemet membrane endothelial keratoplasty. Am J Ophthalmol. 2009;148(2):227-34.

39. Choo SY, Zahidin AZ, Then KY. Re: Spontaneous corneal clearance despite graft detachment in descemet membrane endothelial keratoplasty. Am J Ophthalmol. 2010;149(3):531.

40. Srivastava VK, Singh A, Chowdhary R. Spontaneous resolution of corneal oedema after inadvertent descemetorhexis during cataract surgery. Med J Armed Forces India. 2010;66(2):177-9.

41. Shah RD, Randleman JB, Grossniklaus HE. Spontaneous corneal clearing after Descemet's stripping without endothelial replacement. Ophthalmology. 2012;119(2):256-60.

42. Dirisamer M, Ham L, Dapena I, van Dijk K, Melles GR. Descemet membrane endothelial transfer: "free-floating" donor descemet implantation as a potential alternative to "keratoplasty". Cornea. 2012;31(2):194-7.

43. Koenig SB. Long-term corneal clarity after spontaneous repair of an iatrogenic descemetorhexis in a patient with Fuchs dystrophy. Cornea. 2013;32(6): 886-8.

44. Borkar DS, Veldman P, Colby KA. Treatment of Fuchs endothelial dystrophy by descemet stripping without endothelial keratoplasty. Cornea. 2016;35(10):1267-73.

45. Rao R, Borkar DS, Colby KA, Veldman PB. Descemet membrane endothelial keratoplasty after failed descemet stripping without endothelial keratoplasty. Cornea. 2017;36(7):763-6.

46. Bleyen I, Saelens IE, van Dooren BT, van Rij G. Spontaneous corneal clearing after descemet's stripping. Ophthalmology. 2013;120(1):215.

47. Koenig SB. Planned descemetorhexis without endothelial keratoplasty in eyes with Fuchs corneal endothelial dystrophy. Cornea. 2015;34(9): 1149-51. 
48. Moloney G, Petsoglou C, Ball M, et al. Descemetorhexis without grafting for Fuchs endothelial dystrophy-supplementation with topical ripasudil. Cornea. 2017;36(6):642-8.

49. Huang MJ, Kane S, Dhaliwal DK. Descemetorhexis without endothelial keratoplasty versus DMEK for treatment of Fuchs endothelial corneal dystrophy. Cornea. 2018;37(12):1479-83.

50. Macsai MS, Shiloach M. Use of topical Rho kinase inhibitors in the treatment of Fuchs dystrophy after Descemet stripping only. Cornea. 2019;38(5): 529-34.

51. Davies E, Jurkunas U, Pineda R. Predictive factors for corneal clearance after descemetorhexis without endothelial keratoplasty. Cornea. 2018;37(2): 137-40.

52. Artieda JA, Wells M, Devasahayam RN, Moloney G. 5 -Year outcomes of descemet stripping only in Fuchs dystrophy. Cornea. 2020;39:1048.
53. Okumura N, Okazaki Y, Inoue R, et al. Effect of the rho-associated kinase inhibitor eye drop (ripasudil) on corneal endothelial wound healing. Investig Ophthalmol Vis Sci. 2016;57(3):1284-92.

54. Okumura N, Kinoshita S, Koizumi N. Application of rho kinase inhibitors for the treatment of corneal endothelial diseases. J Ophthalmol. 2017;2017: 2646904.

55. Okumura N, Koizumi N, Ueno $\mathrm{M}$, et al. ROCK inhibitor converts corneal endothelial cells into a phenotype capable of regenerating in vivo endothelial tissue. Am J Pathol. 2012;181(1): 268-77.

56. Kinoshita S, Koizumi N, Ueno M, et al. Injection of cultured cells with a ROCK inhibitor for bullous keratopathy. N Engl J Med. 2018;378(11):995-1003. 